\title{
Effects of Restraint Stress and Nitric Oxide Synthase Inhibition on Learning and Strategy Preference in Young Adult Male Rats
}

\author{
Melih Dağdeviren ${ }^{1}$, Yusuf Hakan Doğan², Lütfiye Kanıt ${ }^{3}$ \\ ${ }^{1}$ Department of Biology, Faculty of Science, Ege University, Izmir, Turkey \\ ${ }^{2}$ Gazikent Family Health Center, Izmir, Turkey \\ ${ }^{3}$ Department of Physiology, Faculty of Medicine, Ege University, İzmir, Turkey
}

\begin{abstract}
Objective: The aim of this study was to investigate the effects of restraint stress and nitric oxide synthase (NOS) inhibition by N $\omega$ Nitro-L-Arginine (LNA) on learning and strategy preference.
\end{abstract}

Material and Methods: Rats were randomly divided into four groups (Saline, Saline+Stress, LNA, LNA+Stress). Stress was applied for one hour in glass cylinders during 13 days. One hour after this stress application, water maze experiments were started. Injections (saline $1 \mathrm{ml} / \mathrm{kg}$ or $50 \mathrm{mg} / \mathrm{kg} \mathrm{LNA}$ ) were given 10 minutes before each experiment. The platform was kept visible or hidden (on the $4^{\text {th }}, 8^{\text {th }}, 12^{\text {th }}$ days) at the same position. On the $13^{\text {th }}$ day the platform was located on the opposite quadrant.

Results: Saline groups exhibited significantly better performances $\left(F_{(1.31)}=174.038 p<0.05\right)$ at the beginning compared to the NOS inhibited groups. For initial hidden platform days; stress was determined as an impairment factor $\left(F_{(1.31)}=5.190 p=0.012\right)$. At the end, acquisition occurred on both visible and hidden platform days for all groups. There was no significant strategy preference difference between the groups.Development of the stress and NOS inhibition impairments were seen, particularly at different periods of the acquisition.

Conclusion: NOS inhibition did not worsen restraint stress-induced learning impairments in rats. Lack of effect may be explained by the antidepressive consequences of NOS inhibition.

Key Words: Learning, NOS inhibition, restraint stress, strategy preference, water maze

Received: 30.08.2011 Accepted: 15.10.2011

\section{Introduction}

Chronic stress reveals a number of physiological responses including glucocorticoids, which reverse the homeostasis of the subject. Animals can exhibit several behavioral responses in stressful conditions according to their emotional perception of the stress. Exposure to chronic stressors can cause long-term structural and functional deficits in the brain, such as reduced memory processing. Hippocampal formation is sensitive to these effects (1).

Place learning in the Water Maze (WM) is a frequently used cognitive test which can be applied with various modifications. Different strategies of learning such as visual, spatial, and response learning can be adapted to the WM apparatus. The WM can also be designed to include more than one option, such as visual or spatial clues, for the animal to solve the problem. This allows researchers to test the cognitive strategy preference (2).

Nitric Oxide Synthase (NOS) is an enzyme that produces nitric oxide (NO) from L-Arginine. NO is a well known gaseous secondary messenger. It produces neurotransmission via $3^{\prime} 5^{\prime}$-cyclic guanosine monophosphate (cGMP) and glu- tamatergic N-methyl-D-Aspartate (NMDA) receptors of surrounding cells. In the central nervous system, NO acts as a retrograde messenger in the glutamatergic NMDA receptor pathway. In the presynaptic terminal, the soluble Guanylate Cyclase ( $\mathrm{sGC}$ ) is induced by NO released from postsynaptic cells. Then secondary messenger cGMP is formed by sGC, and the increasing level of cGMP accelerates the release of glutamate from the presynaptic terminal. As a result, these presynaptic mechanisms contribute to the early phase of long term potentiation (LTP). Thus NO takes part in hippocampal LTP, learning and memory formation processes. For this reason NOS has an important role in these cognitive functions $(3,4)$.

Nitric Oxide Synthase has various forms: The constitutive forms, endothelial NOS (eNOS) and neuronal NOS (nNOS), are found in the brain. On the other hand, inducible NOS (iNOS) is found in immune system cells. Both eNOS and nNOS play a role in cognitive functions (5). NOS inhibition produces impairment in spatial learning and reduces performance in memory tasks (6). N $\omega$ Nitro-LArginine (LNA) is a selective inhibitor on $\mathrm{nNOS}$ and eNOS but not on iNOS (7). 
The relationship between NOS and stress depends on the role of NO in pathological and physiological alterations during hippocampal responses to the stress. Stress-induced changes in nNOS expression levels have also been revealed (8). In some studies it was shown that NOS inhibition has antidepressive effects in rats under stress $(9,10)$. Nevertheless, little has been learned about the relevance of NOS inhibition and stress on learning. The purpose of this study was to investigate the effects of stress and NOS inhibition on cognitive learning abilities and strategy preference in rats by the WM task.

\section{Material and Methods}

\section{Experimental Animals}

Thirty-five male Sprague-Dawley rats (3-4 months old $220 \pm 40$ g) were divided into four groups randomly: Control (Saline, $n=9$ ), Stress (Saline+Stress, $n=9$ ), NOS inhibition (LNA, $n=9$ ), NOS inhibition combined with Stress (LNA+Stress, $n=8$ ). Animals were kept under standard colonial conditions (4/5 animal per cage, $21 \pm 1^{\circ} \mathrm{C}, 12$ hours day/night cycle), food and water were available ad lib. Handling was done for each animal starting three days prior to and during the experiments. Animals were treated according to the European Communities Council directive (86/609/EEC). Ege University Animal Ethics Committee approved this study.

\section{Morris Water Maze Apparatus}

A circular pool (130 cm Ø, $75 \mathrm{~cm}$ in height) was filled with water to a depth of $45 \mathrm{~cm}$ at $22 \pm 1{ }^{\circ} \mathrm{C}$. Water was colored by an opaque, non-toxic, water soluble, dark yellow dye. The water maze tank was virtually divided into four quadrants: south (S), west $(\mathrm{W})$, north $(\mathrm{N})$, and east $(\mathrm{E})$. The platform was located on the NE position of the pool. The platform, when visible, protruded $2.5 \mathrm{~cm}$ above the water and when hidden was submerged $2 \mathrm{~cm}$ below water level. The water tank was located in a $3 \times 4 \mathrm{~m}$ room and extramaze (spatial) cues included items such as posters, cages and two researchers.

\section{Monitoring and Recording}

Experiments were recorded by a camera and a video recorder. Images were captured by a tracker (HVS Image, UK) and processed by a computer with HVS-Water software. Escape latency (EC), path length ( $P L)$, swim speed (SS), and, on probe day, time spent in the quadrant where the platform had been during acquisition (TS) were recorded and analyzed.

\section{Stress protocol}

Restraint stress was applied in glass cylinders $(6.5 \mathrm{~cm} \varnothing, 15$ $\mathrm{cm}$ in height) for one hour (8.30 am-9.30 am) during 13 days. Cylinders were designed to help ventilation but restrict mobility. After stress application, animals were taken to cages and after a one hour recess water maze experiments were started.

\section{Chemical Treatment}

Each day, 10 minutes prior to swim tests, N $\omega$ Nitro-Larginine (LNA, $50 \mathrm{mg} / \mathrm{kg}$ Sigma 5501) or saline $(1 \mathrm{~mL} / \mathrm{kg})$ was injected intraperitoneally to the animals. Non-LNA rats were injected with the same volume of saline as control.

\section{Experimental Protocol}

In 1994 McDonald and White (11) developed a new experimental protocol for water maze that allows learning using visual and navigational cues. Kanit et al. (2) modified this protocol to research cognitive preferences.

Before the first trial, animals were placed on the platform to familiarize the location. Animals were then released sequentially from $\mathrm{S}$ point, allowed to find the platform and then taken to their cage, with at least 15 minutes between releases. Each animal was released from W-N-E points on the same day as done for $\mathrm{S}$ point. If an animal failed to find the platform in 30 seconds during the first trial of the first day, introducing was done. Introducing is accompanying an animal to the platform. Every day the starting point shifted in the clockwise direction. On the $1^{\text {st }}$ to $3^{\text {rd }}, 5^{\text {th }}$ to $7^{\text {th }}, 9^{\text {th }}$ to $11^{\text {th }}$ days, the platform was visible and on the $4^{\text {th }}, 8^{\text {th }}$ and $12^{\text {th }}$ days the platform was hidden. During the whole experiment each animal was released 4 times each day, with either the visible or hidden platform.

On the $13^{\text {th }}$ day, the probe trial was done by placing the platform visibly in the opposite direction (SW direction) to the initial location, and animals were tested for their strategy preference. Animals were released four times and intertrial intervals were at least 15 minutes for the probe trial. Only the data from first releases were assessed for the probe trial, because only this data can test the preference between a learned place and a novel, closer visible platform.

\section{Statistical analysis}

The acquisition of place learning was evaluated by repeated measures analysis of variance (ANOVA) with EL, PL, SS as the dependent variables, and NOS inhibition, stress and days of testing as between and within subjects factors, respectively. Multifactorial and one way ANOVA was performed for probe trial dates and post-hoc analyses were applied as required. SPSS 17.0 program was used for all statistical analyses.

\section{Results}

Three main parameters were recorded, processed and analyzed during the acquisition phase. $E L$ is the time required by an animal to find the platform and is expressed in seconds. PL is the distance covered by an animal to reach the platform and is expressed in meters. SS is the speed of an animal and is expressed in meters per second. EL and PL are the two primary candidate measures for acquisition, however SS gives an idea about non-cognitive abilities or locomotor activity.

To detect the cognitive strategy preference, four parameters were recorded, processed and analyzed for the probe trial. EL, PL and SS parameters and also the percentage of time spent in the quadrant where platform was initially located (TS), for the first release of the first trial of probe day were recorded.

\section{Escape Latencies}

Visible Platform: EL of all groups to find the platform decreased through days, thereby a main effect of days $\left[F_{(1.31)}=39.499 p<0.001\right]$ was observed. These results revealed that all groups of animals learned to find the platform and, at 
EL

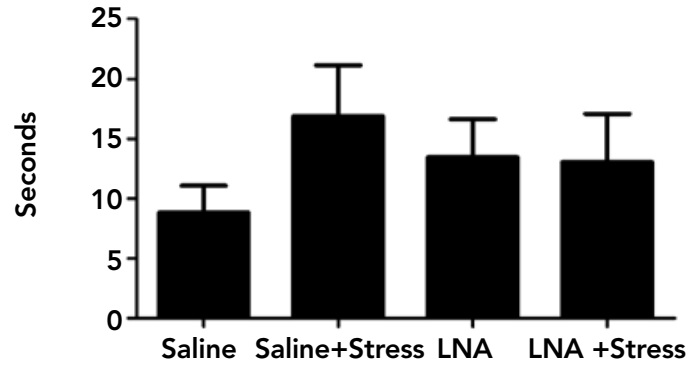

TS

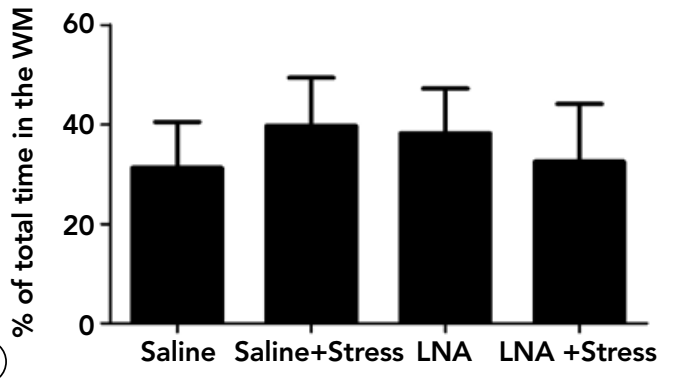

Figure 1. (A) On day 13, trial 1 The escape latency to the new platform and (B) percent time spent in the quadrant where the old platform had been during acquisition. Bars represent group averages \pm SEM. Detailed statistical analyses are given in Section 3

the end of the study, all rats reached asymptotic level. NOS inhibition statistically significantly impaired the acquisition at the early phase when compared to control groups $\left[F_{(1.31)}=174.038\right.$ $\mathrm{p}<0.05]$. There was no statistically significant stress effect or any interaction of the other factors.

Hidden Platform: All groups learned to find the hidden platform's location rapidly $\left[F_{(1.31)}=8.404 p=0.001\right]$. Stress statistically significantly arose as an impairment factor at early phase of hidden platform $\left[F_{(1.31)}=5.190 p=0.012\right]$. NOS inhibition did not have any significant effect in the hidden platform days.

\section{Path Length}

Visible Platform: In subsequent days, rats used a shorter path to reach the platform $\left[F_{(1.31)}=39.436 p<0.001\right]$. On visible platform days, NOS inhibition statistically significantly impaired the acquisition $\left[F_{(1.31)}=10.176 p<0.01\right]$ but stress had no effect on this factor.

Hidden Platform: Rats learned to find the platform quickly $\left[F_{(1.31)}=9.656 p=0.001\right]$. Stress statistically significantly emerged as an impairment factor on acquisition at the early phase $\left[F_{(1.31)}=4.283 p=0.01\right]$.

\section{Swim Speed}

Days emerged as a statistically significant factor $\left[F_{(1.31)}=4.008 p<0.01\right]$ with a visible platform. There was no significant difference between experimental groups, either with other factors of the visible platform or on any hidden platform date.

\section{Probe Trial}

On probe trial day according to the first releases, the escape latency of the control group was quicker and also the path length was shorter than in other groups; however, there was no significant difference between groups (Figure 1A).

There was also no significant difference between the groups in the time spent in the former quadrant of the platform (Figure 1B). When the swim speed values were evaluated, the control group had a better score, but it was not significantly different.

According to our results, all groups of animals preferred spatial strategy. When the data is examined generally, the control group performed better in finding the new platform loca- tion, but nevertheless there was no significant evidence supporting a difference between groups in strategy preference.

\section{Discussion}

Nitric Oxide Synthase inhibition, due to its physiological effects on the brain and vascular endothelial system, can induce a group of responses ranging from altering acquisition and cognitive functions to regulation of stress-related mechanisms. In this study we investigated the effects of restraint stress and NOS inhibition on learning and preference in young adult male rats.

Chronic stress mostly impairs acquisition in the WM, but there is still conflict about it. Some studies show chronic stress can enhance learning in the WM, and some studies demonstrate that there was no effect on learning (12-14). The various results in different studies arose from the different type of chronic stressors, application conditions, and durations. Also the type of data which was evaluated is very important (15). Our results showed that, at the beginning, stress affected rats negatively. On the fourth (first hidden platform) day, which corresponds to the probe day of a classical WM, stress emerged as a significant impairment factor on learning. The stress impaired acquisition at the early phase; was restored mostly on subsequent visible platform days (Figure 2 and 3). It is possible to say that stress precluded conceptual rather than perceptual learning in the initial phase.

N $\omega$ Nitro-L-Arginine was used to inhibit NOS, and LNA does not inhibit iNOS, as aforementioned (7). Scientists showed that nNOS is dramatically dominant to eNOS in the rodent brain (16). But NO involved in LTP is derived from eNOS instead of nNOS (17). NOS inhibition via LNA is appropriate in memory testing experiments. Inhibiting both nNOS and eNOS is essential in order to interrupt acquisition in brain. However, inhibiting eNOS has locomotor effects; non-selective NOS inhibitors increase blood pressure, which can alter performance by changing the muscular functions in learning and memory tests (18). A decrease in motor activity is an inevitable effect of NOS inhibition, especially with nonspecific NOS inhibitors (19). To gauge locomotor activity reduction, swim speed data is an alternative option. As previously reported in a sex difference study, the decrease in swim speed 


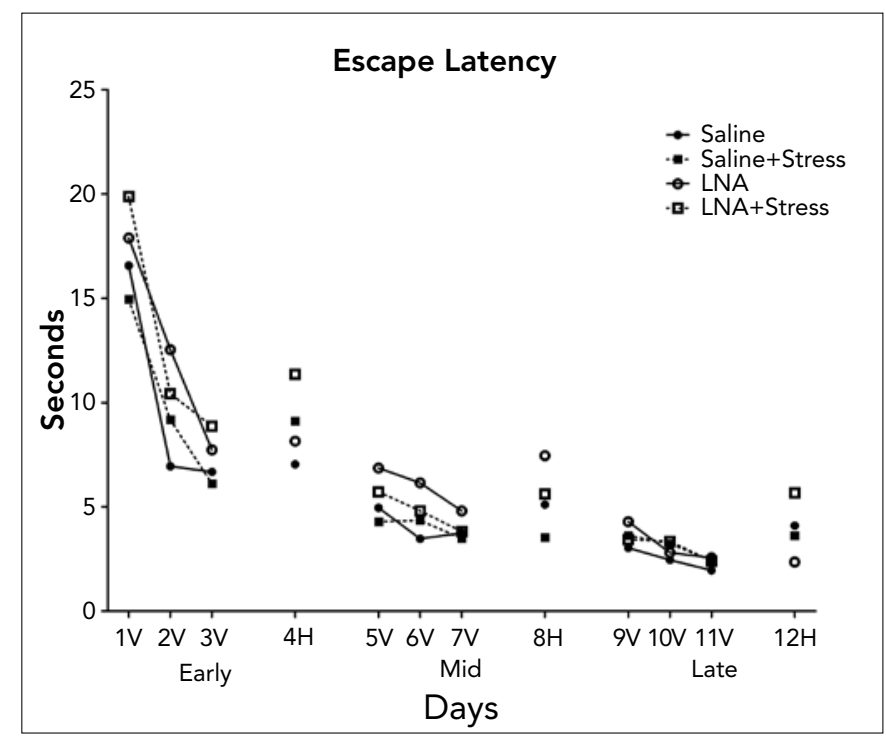

Figure 2. Mean escape latency (EL) during the acquisition of place learning with the platform visible (V) on days 1-3, 5-7, 9-11, and hidden $(H)$ on days 4, 8, 12. Symbols represent group averages of all four trials on each day. Filled circles with solid lines represent the Saline group. Filled squares with dotted lines represent the Saline+Stress group. Open circles with solid lines represent the LNA group. Open squares with dotted lines represent the LNA+Stress group. Detailed statistical analyses are given in Section 3. For visual clarity, the error bars are not included in the figures

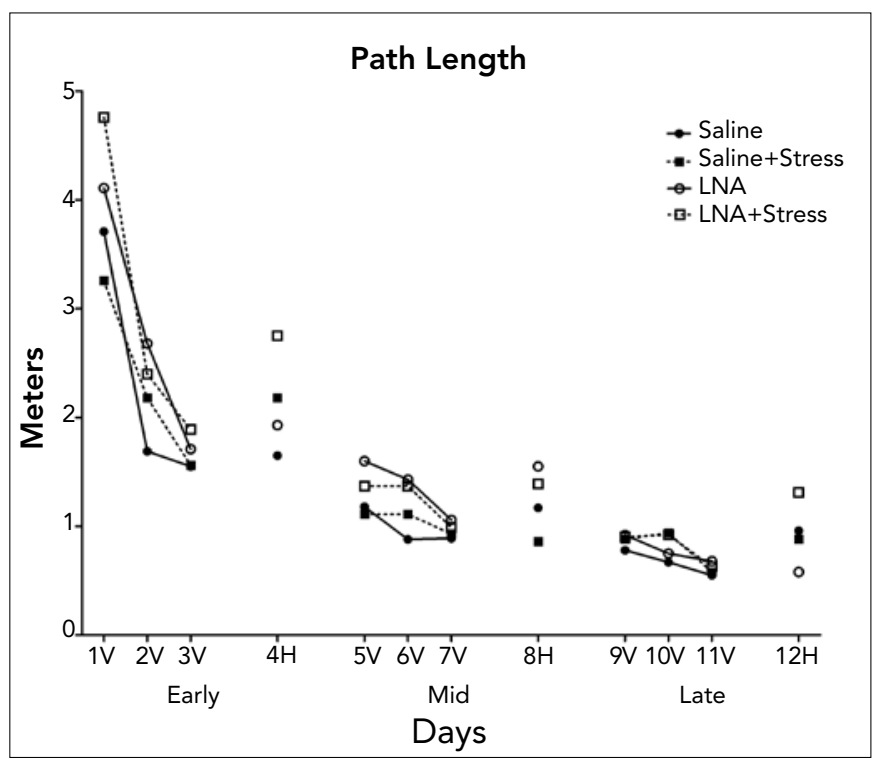

Figure 3. Mean path length $(P L)$ during the acquisition of place learning with the platform visible (V) on days 1-3, 5-7, 9-11, and hidden $(H)$ on days 4, 8, 12. Symbols represent group averages of all four trials on each day. Filled circles with solid lines represent the Saline group. Filled squares with dotted lines represent the Saline+Stress group. Open circles with solid lines represent the LNA group. Open squares with dotted lines represent the LNA+Stress group. Detailed statistical analyses are given in Section 3. For visual clarity, the error bars are not included in the figures after NOS inhibition was apparent mostly in female rats (20). Our results confirm that there was no difference between NOS inhibited and saline-treated male rats. Also, stress did not affect the swim speed. In one study, 7-nitro-indazole was applied to inhibit nNOS selectively, to refrain from decreasing motor activity. The impairment was seen at the early phase, but at the late phase there was no impairment in radial arm maze (18). The results of this selective NOS inhibitor study and our findings are overlapping, which may suggest that the systemic effects of LNA have not biased our results. It was shown in some NOS inhibition studies that LNA has negative effects on acquisition at the early phase $(20,21)$. If the acquisition period was kept between four to seven days, the impairment was observed during the whole experiment $(22,23)$. However in longer acquisition periods, such as 12 days, the impairment gradually disappeared after the middle phase (20). Our results proved that, again, NOS inhibition impaired the acquisition during the earlymid phases and this impairment disappeared at the late phase.

In one study, antinociceptive effects of NOS inhibition emerged five days after the stress application, indicating that stress masked the antinociceptive effect of NOS inhibition for five days (24). Also, in several studies it was shown that NOS inhibition has antidepressive effects on rodents $(9,10,25)$. It was demonstrated that restraint stress affects the NOS dependent nociceptive mechanisms of the hippocampus at the cellular level (8). There may be a strong relationship between NOS inhibition and stress. In our study, there was an increase instead of a decrease in the learning performance of NOS inhibition combined stress group at the beginning of the mid phase. In a knock-out nNOS study it was shown that learning was impaired in the WM and other learning experiments. However, it was concluded that little evidence exists for the antidepressive effects of NOS deficiency. Wultsh et al. (26) claimed that nNOS deficient animals should be used as a model for Alzheimer's disease or attention deficit disorder in order to emphasize the antidepressive effects of NOS inhibition.

Strategy preference may differ from strain to strain or male to female $(2,27)$. In this study, at the late phase of WM there was no difference between groups for the time and distance for reaching the platform (Figure 2 and 3), so we were able to investigate the preference on probe trial. Our results showed that there was no significant difference between groups according to their learning strategy preference (Figure 1). Previously it was displayed that there was a tendency in NOS inhibited male rats to prefer the new visual platform, like females, in strategy learning (20). It is ambiguous that NOS inhibition reveals a female type behavior pattern. According to our findings, there is no tendency toward female type behavior pattern in either the stressed, the NOS inhibited or the NOS inhibition combined stress groups. Because of these results, we suggested that, in NOS inhibition and stress groups, spatial preferences are more prominent than in controls.

\section{Conclusion}

Our findings indicate that stress and NOS inhibition created impairment on acquisition particularly and at different periods. Stress especially impaired acquisition for the first 
hidden platform day. NOS inhibition created impairment at the early phase of visible platform days. In conclusion, NOS inhibition does not amplify the impairment created by chronic restraint stress and this may be a result of antidepressive effects of NOS inhibition.

\section{Acknowledgements}

This study was supported by the "Center for Brain Research" unit of Ege University. The authors are grateful to Jacki Gutenkunst Gozen; English instructor at Izmir University of Economics, for editing and correcting the manuscript.

\section{Conflict of Interest}

No conflict of interest was declared by the authors.

\section{References}

1. Kumar RS, Narayanan SN, Nayak S. Ascorbic acid protects against restraint stress-induced memory deficits in wistar rats. Clinics (Sao Paulo) 2009;64:1211-7. [CrossRef]

2. Kanit L, Taskiran D, Furedy JJ, Kulali B, McDonald R, Pogun S. Nicotine interacts with sex in affecting rat choice between "lookout" and "navigational" cognitive styles in the Morris water maze place learning task. Brain Res Bull 1998;46:441-5. [CrossRef]

3. Domek-Lopacinska KU, Strosznajder JB. Cyclic GMP and nitric oxide synthase in aging and Alzheimer's disease. Mol Neurobiol 2010;41:129-37. [CrossRef]

4. Hawkins RD, Son H, Arancio O. Nitric oxide as a retrograde messenger during long-term potentiation in hippocampus. Prog Brain Res 1998;118:155-72. [CrossRef]

5. Paakkari I, Lindsberg P. Nitric oxide in the central nervous system. Ann Med 1995;27:369-77. [CrossRef]

6. Ingram DK, Spangler EL, Meyer RC, London ED. Learning in a 14-unit T-maze is impaired in rats following systemic treatment with N omega-nitro-L-arginine. Eur J Pharmacol 1998;341:1-9. [CrossRef]

7. Lambert LE, Whitten JP, Baron BM, Cheng HC, Doherty NS, McDonald IA. Nitric oxide synthesis in the CNS endothelium and macrophages differs in its sensitivity to inhibition by arginine analogues. Life Sci 1991;48:69-75. [CrossRef]

8. Echeverry MB, Guimaraes FS, Del Bel EA. Acute and delayed restraint stress-induced changes in nitric oxide producing neurons in limbic regions. Neuroscience 2004;125:981-93. [CrossRef]

9. Sevgi S, Ozek M, Eroglu L. L-NAME prevents anxiety-like and depression-like behavior in rats exposed to restraint stress. Methods. Find Exp Clin Pharmacol 2006;28:95-9. [CrossRef]

10. Ulak G, Mutlu O, Akar FY, Komsuoglu FI, Tanyeri P, Erden BF. Neuronal NOS inhibitor 1-(2-trifluoromethylphenyl)-imidazole augment the effects of antidepressants acting via serotonergic system in the forced swimming test in rats. Pharmacol Biochem Behav 2008;90:563-8. [CrossRef]

11. McDonald RJ, White NM. Parallel information processing in the water maze: evidence for independent memory systems involving dorsal striatum and hippocampus. Behav Neurol Biol 1994;61:260-70. [CrossRef]

12. Kitraki E, Kremmyda O, Youlatos D, Alexis MN, Kittas C. Genderdependent alterations in corticosteroid receptor status and spa- tial performance following 21 days of restraint stress. Neuroscience 2004;125:47-55. [CrossRef]

13. Li XH, Liu NB, Zhang MH, Zhou YL, Liao JW, Liu XQ, et al. Effects of chronic multiple stress on learning and memory and the expression of Fyn, BDNF, TrkB in the hippocampus of rats. Chin Med J (Engl) 2007;120:669-74.

14. Venero C, Tilling T, Hermans-Borgmeyer I, Schmidt R, Schachner $M$, Sandi C. Chronic stress induces opposite changes in the mRNA expression of the cell adhesion molecules NCAM and L1. Neuroscience 2002;115:1211-9. [CrossRef]

15. Conrad CD. A critical review of chronic stress effects on spatial learning and memory. Prog Neuropsychopharmacol Biol Psychiatry 2010;34:742-55. [CrossRef]

16. Hara $H$, Waeber $C$, Huang $P L$, Fuiji M, Fishman MC, Moskowitz MA. Brain distribution of nitric oxide synthase in neuronal or endothelial nitric oxide synthase mutant mice using [3H]L-NGnitro-arginine autoradiography. Neuroscience 1996;75:881-90. [CrossRef]

17. O'Dell TJ, Huang PL, Dawson TM, Dinerman JL, Snyder SH, Kandel ER, et al. Endothelial NOS and the blockade of LTP by NOS inhibitiors in mice lacking neuronal NOS. Science 1994;265:542-6. [CrossRef]

18. Holscher C, McGlinchey L, Anwyl R, Rowan MJ. 7-nitro indazole, a selective neuronal nitric oxide synthase inhibitor in vivo, impairs spatial learning in the rat. Learn Mem 1996;2:267-78. [CrossRef]

19. Sandi C, Venero C, Guaza C. Decreased spontaneous motor activity and startle response in nitric oxide synthase inhibitor-treated rats. Eur J Pharmacol 1995;227:89-97. [CrossRef]

20. Kanit L, Koylu E, Yararbas G, Furedy JJ, Pogun S. The effect of nitric oxide synthase inhibition on cognitive ability and strategies employed for place learning in the water maze: sex differences. Brain Res Bull 2003;62:151-9. [CrossRef]

21. Mogensen J, Wortwein G, Hasman A, Nielsen P, Wang Q. Functional and neurochemical profile of place learning after L-nitro-arginine in the rat. Neurbiol Learn Mem 1995;63:54-65. [CrossRef]

22. Koylu EO, Kanit L, Taskiran D, Dagci T, Balkan B, Pogun S. Effects of nitric oxide synthase inhibition on spatial discrimination learning and central DA2 and mACh receptors. Pharmacol Biochem Behav 2005;81:32-40. [CrossRef]

23. Maljessi N, Kadkhodaee M, Parviz M, Nagdhi N. Serotonin depletion in rat hippocampus attenuates L-NAME-induced spatial learning deficits. Brain Res 2003;963:244- 51. [CrossRef]

24. Echeverry MB, Guimaraes FS, Oliveira MA, do Prado WA, Del Bel EA. Delayed stress-induced antinociceptive effect of nitric oxide synthase inhibition in the dentate gyrus of rats. Pharmacol Biochem Behav 2002;74:149-56. [CrossRef]

25. Harkin A, Connor TJ, Burns MP, Kelly JP. Nitric oxide synthase inhibitors augment the effects of serotonin re-uptake inhibitors in the forced swimming test. Eur Neuropsychopharmacol 2004;14:274-81. [CrossRef]

26. Wultsch T, Chourbaji S, Fritzen S, Kittel S, Grunblatt E, Gerlach M, et al. Behavioral and expressional phenotyping of nitric oxide synthase-I knockdown animals. J Neural Transm Suppl 2007;72:69-85. [CrossRef]

27. Sung JY, Goo JS, Lee DE, Jin DQ, Bizon JL, Gallagher M, et al. Learning strategy selection in the water maze and hippocampal CREB phosphorylation differ in two inbred strains of mice. Learn Mem 2008;15:183-8. [CrossRef] 